\title{
Association between secondhand smoke exposure and early eruption of deciduous teeth: A cross-sectional study
}

\author{
Takashi Hanioka', Miki Ojima², Keiko Tanaka ${ }^{3}$, Nao Taniguchi', Kaoru Shimada ${ }^{4}$, Takeshi Watanabe
}

\begin{abstract}
INTRODUCTION Secondhand smoke (SHS) exposure is a risk factor for early childhood caries. Here we examined the association between SHS exposure and early tooth eruption (ETE) to clarify the additional etiology of an increased chance of contact between the tooth's surface and acid produced by fermenting oral bacteria.

METHODS Data of 388 child-mother pairs who attended health checkups at public health centers were assessed for children aged $\geqslant 18$ months. SHS exposure was reported as maternal smoking during pregnancy and household smoking after birth. Associations between SHS exposure and ETE ( $\geqslant 3$ canines in the oral cavity) were tested using multivariable analyses of the dose-response relationship. Subgroup and sensitivity analyses were performed for birth-weight subgroups and SHS exposure variables, respectively.

RESULTS ETE prevalence was $65.5 \%, 68.1 \%$, and $76.9 \%$ in the no, medium-dose (ceased partway and sometimes), and highest-dose (every day) exposure groups, respectively, during pregnancy, and $61.5 \%, 75.0 \%$, and $75.5 \%$, respectively, after birth. The association between the highest dose exposure during pregnancy and ETE was not significant $(\mathrm{OR}=1.42,95 \%$ CI: $0.34-5.96, \mathrm{p}=0.631)$, whereas that between highest dose exposure after birth and ETE was significant $(\mathrm{OR}=2.13,95 \%$ CI: $1.06-4.31, \mathrm{p}=0.034)$; this association was distinct in the subgroup of children with smaller birth weights $(<3000 \mathrm{~g})(\mathrm{OR}=3.19,95 \% \mathrm{CI}: 1.08-9.44, \mathrm{p}=0.036)$. The dose-response relationship was consistently significant for exposure after birth $(\mathrm{p}<0.05)$. The sensitivity analysis that employed no SHS exposure, as a reference, revealed that exposure after birth but no exposure during pregnancy was significantly associated with ETE (OR=2.29, 95\% CI: $1.19-4.40, \mathrm{p}=0.013$ ). However, the association between exposure during pregnancy and ETE was consistently non-significant ( $\mathrm{p}>0.05$ ).

CONCLUSIONS When controlling for variables of birth weight and exposure type, SHS exposure after birth was independently associated with the early eruption of deciduous canines. Further studies are warranted to examine the trajectory of SHS exposure after birth, ETE, and early childhood caries incidence.
\end{abstract}

\author{
AFFILIATION \\ 1 Department of Preventive \\ and Public Health Dentistry, \\ Fukuoka Dental College, \\ Fukuoka, Japan \\ 2 Department of Oral Health \\ Sciences, Faculty of Nursing \\ and Health Care, BAIKA \\ Women's University, Osaka, \\ Japan \\ 3 Department of \\ Epidemiology and Preventive \\ Medicine, Ehime University \\ Graduate School of Medicine, \\ Ehime, Japan \\ 4 Department of Nursing, \\ Faculty of Nursing, Fukuoka \\ Nursing College, Fukuoka, \\ Japan
}

CORRESPONCENCE TO:

Takashi Hanioka. Department of Preventive and Public Health Dentistry, Fukuoka Dental College, 2-15-1, Tamura, Sawara-ku, 814-0193

Fukuoka, Japan.

Email:haniokat@college.

fdenet.ac.jp

KEYWORDS

tooth eruption, deciduous tooth, secondhand smoke, maternal smoking, household smoking

Received: 8 November 2017 Revised: 31 December 2017 Accepted: 29 January 2018

\section{INTRODUCTION}

Secondhand smoke (SHS) exposure causes significant morbidity and mortality in children ${ }^{1}$, and adversely influences oral health in addition to respiratory health ${ }^{2,3}$. Maternal smoking exposure is associated with congenital defects of the orofacial cleft $^{4}$, whereas SHS 
exposure is associated with early childhood caries ${ }^{4-6}$. Although deciduous teeth are eventually replaced with permanent teeth, early childhood caries are a public health problem, because they are associated with toothache, mastication, orofacial development, and permanent tooth alignment, in children.

The primary etiology of dental caries is cariogenic contact between premature enamel crystals of erupting teeth and acid produced by fermenting oral bacteria harboring on a tooth's surface ${ }^{7}$. Tobacco smoke exposure increases the susceptibility to acid because: calcification of the enamel matrix is inhibited ${ }^{8}$, enamel crystals grow prematurely ${ }^{9}$, and acid-producing plaques are enhanced ${ }^{10,11}$ on developmental defects of the tooth's surface ${ }^{12}$. Early tooth eruption (ETE) may be added to the etiology of dental caries because ETE in the oral cavity could increase the likelihood of cariogenic contact ${ }^{13}$.

However, the association between SHS exposure during pregnancy after birth and early childhood caries remains unclear. An additive effect of prenatal maternal smoking and postnatal SHS exposure on early childhood caries was observed ${ }^{14}$. The hazard ratio for early childhood caries between cases of maternal smoking during pregnancy and those of no smoking in the family was 1.10 in a cohort of 12 729 children, while the associated risk from tobacco smoke exposure after birth increased by $2.14^{15}$. Similarly, studies that assessed the association between the timing of tooth eruption and SHS exposure, including maternal smoking, have reported inconsistent findings ${ }^{16-18}$, with one study reporting no significant effect ${ }^{17}$. The first deciduous tooth eruption occurred approximately 1 week earlier in children who were exposed to maternal smoking during pregnancy compared with those who were not exposed $^{16}$, and deciduous tooth eruption negatively correlated with maternal smoking exposure during pregnancy $^{18}$. Furthermore, low birth weight of $<2500$ $\mathrm{g}$ is associated with delayed tooth eruption ${ }^{19}$, and premature birth is associated with SHS exposure ${ }^{1}$.

Understanding the association between SHS exposure and early childhood caries with regard to the timing of exposure and interaction with birth weight is difficult. Thus, clarifying the potential association between SHS exposure and ETE may help to explaining the effect of such an exposure on increased susceptibility to early childhood caries.
Here, we assessed the association between SHS exposure and ETE of deciduous teeth with regard to birth weight and timing of exposure.

\section{METHODS}

\section{Study population}

In Japan, periodic health checkups for infants at public health centers are required, by law, to include oral health examinations at the age of 18-23 months; but most children undergo checkups at the age of 18 or 19 months. On the basis of the smoking rate of mothers of 18-month-old children and the expected rate of dropout, a sample size of 450 was considered to be appropriate. The survey was performed from March to September 2014 at public health centers in three cities in Shizuoka Prefecture. Permission for conducting this present study was obtained from the local government of each city. The study protocol was approved by the Ethics Committee of the Fukuoka Dental College (Ethics Approval No. 224).

\section{Data collection}

The mailed notices regarding health checkups of 485 children included a questionnaire and an informed consent form. Addresses were obtained from the basic resident register according to the date of birth. Written informed consent was obtained from the guardians of all children. Health records and questionnaires that were completed by the children's guardians were used to assess the association between SHS exposure and ETE. Other data of the enrolled children, such as existing teeth, were transcribed from the health records after oral examination. The dentists who usually examined children at the public health centers were notified of the study objective in advance but were blinded to the children's exposure status to reduce the information bias of the examiners.

\section{Outcome measure of ETE}

In the study population, $\geqslant 50 \%$ of the children had 16 teeth, and almost no children had four second-deciduous molars. In general, except for the second deciduous molars, canines are the last teeth to emerge in the oral cavity of children at the age of 18-23 months. Therefore, the number of canines was used as a measure of ETE. In this study, the presence of three or four canines in the oral cavity was defined as ETE. Furthermore, emergence of at least one part of the tooth structure in the oral 
cavity was defined as an existing tooth, and fused teeth were recorded as two independent teeth. Because this definition of an existing tooth is universal in Japan, no calibration among the dentists was performed. The ETE variable was dichotomized as a dependent variable for statistical analyses.

\section{Assessment of SHS exposure}

Data concerning each child's SHS exposure were obtained on the basis of two questions that assessed maternal smoking during pregnancy and household smoking around the child after birth. In addition, data regarding the use of other tobacco products and exposure to thirdhand smoke were excluded because such products and concepts were not popular in Japan at the time of survey. For each question, the mother selected oneresponsefrom thefollowingfourcategories: no, sometimes, every day, and ceased partway. Because the number of children in the 'sometimes' and 'ceased partway' categories of SHS exposure was small for statistical analyses, these categories were combined into a single exposure category that comprised the medium-dose exposure category.

\section{Potential confounders}

Gender (male or female), location of residence (city A, B, or C), birth order (first or other than first), and parents' education level (no more than high school or college level or above; the higher level was recorded in cases where the parents' education level differed), and annual income $(<3,3-4.9$, or $\geqslant 5$ million yen) were included in the statistical model as covariates of categorical variables, and the first category of each variable was used as reference. According to the monthly average in March 2014, one million yen was equivalent to 9778 US dollars. Age and birth weight were included in the model as covariates of continuous variables. Data regarding the variables of age, birth order, and birth weight of children living in city A were transcribed from health records, whereas those for children living in cities B and C were obtained from the questionnaire.

\section{Statistical analysis}

Datasets with no missing values were used for the multivariable analyses. Chi-squared test and oneway ANOVA were used for bivariate analyses. Multivariable logistic regression analyses were performed to independently test the association between SHS exposure and ETE, when all independent variables were included. Children were divided into two subgroups according to birth weight $(<3000 \mathrm{~g}$ and $\geqslant 3000 \mathrm{~g})$. Each group was subjected to a subgroup analysis wherein birth weight was included as a continuous variable. Trends were analyzed by incorporating SHS exposure variables as ordinal variables. For sensitivity analyses, children not exposed to maternal smoking during pregnancy or household smoking after birth were used as reference. For the maternal smoking question, the no exposure group was divided into 'no exposure' and 'exposure to household smoking only' groups. For the household smoking question, the no exposure group was divided into 'no exposure' and 'exposure to maternal smoking during pregnancy only' groups. These analyses were conducted using statistical software (IBM SPSS 20.0; IBM Corp., New York, NY, USA), with the significance level set at $5 \%$.

\section{RESULTS}

\section{Characteristics regarding SHS exposure}

Data of 430 children ( $88.7 \%$ of invited children) were collected. Finally, datasets of 388 (80.0\%) pairs without values that were either missing or incorrect were used for the analyses. The distribution of children according to the questions regarding SHS exposure is summarized in a contingency table (Table 1). Most children (63.4\%) were not exposed to SHS. Maternal smoking exposure during pregnancy and household smoking exposure after birth were documented in

\section{Table 1. Contingency table of SHS exposure variables}

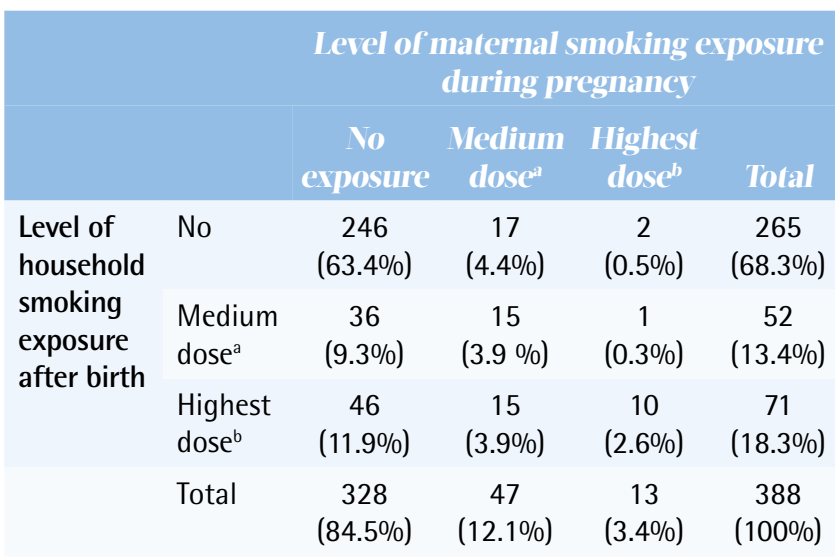

The association between two measures of exposure was significant at $p<0.001$ ( $x^{2}$ test) Two cells $(22.2 \%)$ had an expected frequency of $<5$. Category of questionnaire:

a 'sometimes and ceased partway' and ' 'every day'. 
$15.5 \%$ and $31.7 \%$ of children, respectively.

Characteristics of children according to SHS exposure and ETE

Table 2 shows the distribution and characteristics of children according to SHS exposure levels that were stratified based on potential confounders. For the categorical variables, significant negative associations were observed between both the types of SHS exposure and parents' education level and annual income, whereas gender, location of residence, and birth order were not significantly associated with

Table 2. Distribution and characteristics of children according to two measures of SHS exposure stratified based on potential confounders

\begin{tabular}{|c|c|c|c|c|c|c|c|}
\hline \multicolumn{8}{|c|}{ SIIS exposure levels } \\
\hline \multirow[t]{2}{*}{ Variables and categories } & \multicolumn{3}{|c|}{ Vaternal smoking during pregnancy } & \multicolumn{3}{|c|}{ Houschold smoking after birth } & \multirow[t]{2}{*}{ Total } \\
\hline & No exposure & $\begin{array}{c}\text { Medium } \\
\text { dose }^{\mathrm{a}}\end{array}$ & $\begin{array}{c}\text { Highest } \\
\text { dose }^{b}\end{array}$ & No exposure & $\begin{array}{c}\text { Medium } \\
\text { dose }^{\mathrm{a}}\end{array}$ & $\begin{array}{c}\text { Highest } \\
\text { dose }^{b}\end{array}$ & \\
\hline \multicolumn{8}{|c|}{ Number (\%) for categorical variables } \\
\hline \multicolumn{8}{|l|}{ Gender } \\
\hline Male & $165(83.3)$ & $26(13.1)$ & $7(3.5)$ & $133(67.2)$ & $28(14.1)$ & $37(18.7)$ & 198(100) \\
\hline \multirow[t]{2}{*}{ Female } & $163(85.8)$ & $21(11.1)$ & $6(3.2)$ & $132(69.5)$ & $24(12.6)$ & $34(17.9)$ & $190(100)$ \\
\hline & & $p=0.796$ & & \multicolumn{4}{|c|}{$p=0.872$} \\
\hline \multicolumn{8}{|l|}{ Location of residence } \\
\hline A city & $55(80.9)$ & $10(14.7)$ & $3(4.4)$ & $49(72.1)$ & $5(7.4)$ & $14(20.6)$ & $68(100)$ \\
\hline B city & $178(81.7)$ & $30(13.8)$ & $10(4.6)$ & $138(63.3)$ & $35(16.1)$ & $45(20.6)$ & $218(100)$ \\
\hline \multirow[t]{2}{*}{ C city } & $95(93.1)$ & $7(6.9)$ & $0(0)$ & $78(76.5)$ & $12(11.8)$ & $12(11.8)$ & $102(100)$ \\
\hline & & $p=0.060^{c}$ & & \multicolumn{4}{|c|}{$p=0.079$} \\
\hline \multicolumn{8}{|l|}{ Birth order } \\
\hline First & $151(83.9)$ & $21(11.7)$ & $8(4.4)$ & $125(69.4)$ & $21(11.7)$ & $34(18.9)$ & $180(100)$ \\
\hline \multirow[t]{2}{*}{$\geq$ Second } & $177(85.1)$ & $26(12.5)$ & $5(2.4)$ & $140(67.3)$ & $31(14.9)$ & $37(17.8)$ & 208(100) \\
\hline & & $p=0.530$ & & \multicolumn{4}{|c|}{$p=0.643$} \\
\hline \multicolumn{8}{|l|}{ Highest education of parents } \\
\hline$\leq$ High school & $85(69.7)$ & $28(23.0)$ & $9(7.4)$ & $52(42.6)$ & $29(23.8)$ & $41(33.6)$ & $122(100)$ \\
\hline \multirow[t]{2}{*}{$\geq$ College } & $243(91.4)$ & $19(7.1)$ & $4(1.5)$ & $213(80.1)$ & $23(8.6)$ & $30(11.3)$ & $266(100)$ \\
\hline & & $\mathrm{p}<0.001^{\mathrm{d}}$ & & \multicolumn{4}{|c|}{$p<0.001$} \\
\hline \multicolumn{8}{|c|}{ Annual income of parents, million yen } \\
\hline$<3$ & $38(69.1)$ & $14(25.5)$ & $3(5.5)$ & $27(49.1)$ & $13(23.6)$ & $15(27.3)$ & $55(100)$ \\
\hline $3-4.9$ & $166(86.5)$ & $18(9.4)$ & $8(4.2)$ & $123(64.1)$ & $28(14.6)$ & $41(21.4)$ & 192(100) \\
\hline \multirow[t]{2}{*}{$\geq 5$} & $124(87.9)$ & $15(10.6)$ & $2(1.4)$ & $115(81.6)$ & $11(7.8)$ & $15(10.6)$ & $141(100)$ \\
\hline & & $p=0.007^{c}$ & & \multicolumn{4}{|c|}{$\mathrm{p}<0.001$} \\
\hline Total & $328(84.5)$ & $47(12.1)$ & $13(3.4)$ & $265(68.3)$ & $52(13.4)$ & $71(18.3)$ & $388(100)$ \\
\hline \multicolumn{8}{|c|}{ Mean \pm standard deviation for continuous variables } \\
\hline \multirow[t]{3}{*}{ Age, months } & 18.7 & 18.6 & 18.5 & 18.6 & 18.8 & 18.7 & 18.7 \\
\hline & \pm 0.9 & \pm 0.7 & \pm 0.5 & \pm 0.8 & \pm 0.9 & \pm 0.8 & \pm 0.8 \\
\hline & & $p=0.758$ & & & $\mathrm{p}=$ & & \\
\hline \multirow[t]{3}{*}{ Birth weight, $g$} & 2983.9 & 3026.1 & 2707.5 & 2959.7 & 3075.4 & 2984.3 & 2979.7 \\
\hline & \pm 409.1 & \pm 348.2 & \pm 371.3 & \pm 402.9 & \pm 417.1 & \pm 391.3 & \pm 403.6 \\
\hline & & $p=0.037$ & & \multicolumn{4}{|c|}{$p=0.167$} \\
\hline
\end{tabular}




SHS exposure. Among the continuous variables, negative association between birth weight and maternal smoking during pregnancy was significant; whereas associations between birth weight and household smoking after birth, or between age and SHS exposure, were not significant.

ETE prevalence was significantly associated with residence (highest prevalence for city $B$, $p=0.011)$, older age $(p=0.001)$, and higher birth weight $(p=0.020)$, among all potential confounders (data available in appendix table 1). No significant association was observed with other variables.

\section{Association between SHS exposure and ETE}

Overall, ETE prevalence was $66.2 \%$ (Table 3). The trend in the distribution of children with ETE according to the SHS exposure level was different for each type of SHS exposure. For maternal smoking exposure during pregnancy, ETE prevalence was $65.5 \%$ for no exposure, $68.1 \%$ for medium-dose exposure, and $76.9 \%$ for highest-dose exposure. For household smoking exposure after birth, ETE prevalence was 61.5\%, $75.0 \%$, and $77.5 \%$, respectively. The 'no exposure' groups showed the lowest ETE prevalence for both types of exposure. ETE prevalence was consistently highest in the highest-dose exposure group for both types of exposure. Adjusted odds ratios (AORs) and 95\% confidence intervals (CIs) of the highest-dose exposure group were 1.42 , and $0.34-5.96(p=0.631)$, respectively, for maternal smoking exposure during pregnancy, and 2.13, and 1.06-4.31 ( $\mathrm{p}=0.034)$, for household smoking exposure after birth. The association between ETE and SHS exposure and the dose-response relationship were significant only for household smoking exposure after birth.

\section{Subgroup analysis}

Table 4 shows ETE prevalence, and crude and AORs of ETE prevalence according to the SHS exposure level based on exposure type in children with birth weights of $<3000 \mathrm{~g}$. The trend in the distribution of children according to the SHS exposure level was also different for each type of SHS exposure. For the no, medium-dose, and highest-dose exposure groups, ETE prevalence was 58.1\%, 69.6\%, and $72.7 \%$, respectively, for maternal smoking during pregnancy, and $54.2 \%, 68.2 \%$, and $80.0 \%$, respectively, for household smoking after birth. Compared to the AORs of the highest-dose exposure groups of all children (Table 3), that of exposed children to maternal smoking during pregnancy was lower, 0.98 vs 1.42 , whereas that of exposed children to household smoking after birth was higher, 3.19 vs 2.13. The dose-response relationship was negative for maternal smoking exposure during pregnancy. Regarding household smoking exposure after birth, the association and dose-response relationship were again significant ( $\mathrm{p}=0.036$ and 0.019 , respectively). No significant association between SHS exposure and ETE was found in children with birth weights of $\geqslant 3000 \mathrm{~g}$ (data available in appendix table 2 ).

\section{Sensitivity analysis}

Table 5 shows ETE prevalence, crude and AORs of

Table 3. Prevalence of early tooth eruption (ETE) and odds ratios (ORs) and $95 \%$ confidence intervals (CIs) of ETE according to SHS exposure level with regard to exposure type

\begin{tabular}{|c|c|c|c|c|c|c|}
\hline \multirow[b]{2}{*}{ Exposure type } & \multirow[b]{2}{*}{ Exposure level } & \multirow[b]{2}{*}{ ETE \% (n) } & \multicolumn{2}{|c|}{ Crude } & \multicolumn{2}{|c|}{ Adjusted } \\
\hline & & & OR, $95^{\circ} \% \mathrm{CI}$ & p & OR, $95^{\circ} \% \mathrm{CI}$ & p \\
\hline \multirow{4}{*}{$\begin{array}{l}\text { Maternal smoking } \\
\text { during pregnancy }\end{array}$} & No exposure & $65.5(215)$ & Reference & & Reference & \\
\hline & Medium dose ${ }^{\mathrm{a}}$ & $68.1(32)$ & $1.12,0.58-2.16$ & 0.732 & $0.95,0.45-1.98$ & 0.888 \\
\hline & Highest dose $\mathrm{e}^{\mathrm{b}}$ & $76.9(10)$ & $1.75,0.47-6.49$ & 0.402 & $1.42,0.34-5.96$ & 0.631 \\
\hline & & & \multicolumn{2}{|c|}{$p$ for trend $=0.479$} & \multicolumn{2}{|c|}{$p$ for trend $=0.268$} \\
\hline \multirow{5}{*}{$\begin{array}{l}\text { Household smoking } \\
\text { after birth }\end{array}$} & No exposure & $61.5(163)$ & Reference & & Reference & \\
\hline & Medium dose ${ }^{\mathrm{a}}$ & $75.0(39)$ & $1.88,0.96-3.69$ & 0.067 & $1.71,0.80-3.63$ & 0.163 \\
\hline & Highest dose $\mathrm{e}^{\mathrm{b}}$ & $77.5(55)$ & $2.15,1.17-3.96$ & 0.014 & $2.13,1.06-4.31$ & 0.034 \\
\hline & & & \multicolumn{2}{|c|}{$p$ for trend $=0.004$} & \multicolumn{2}{|c|}{$p$ for trend $=0.011$} \\
\hline & Total & $66.2(257)$ & & & & \\
\hline
\end{tabular}

Category of questionnaire: a 'sometimes and ceased partway' and ' 'every day'. 
Table 4. Prevalence of early tooth eruption (ETE) and odds ratios (ORs) and 95\% confidence intervals (CIs) of ETE according to SHS exposure level with regard to exposure type in children with birth weights of $<3000 \mathrm{~g}$

\begin{tabular}{|c|c|c|c|c|c|c|}
\hline \multirow[b]{2}{*}{ Exposure type } & \multirow[b]{2}{*}{ Exposure level } & \multirow{2}{*}{$\begin{array}{c}\text { ETE \% } \\
\text { (n total n) }\end{array}$} & \multicolumn{2}{|c|}{ Crude } & \multicolumn{2}{|c|}{ Adjusted } \\
\hline & & & $\mathrm{OR}, 95^{\circ} \% \mathrm{CI}$ & $p$ & OR, $95^{\circ} \% \mathrm{CI}$ & p \\
\hline \multirow{4}{*}{$\begin{array}{l}\text { Maternal smoking } \\
\text { during pregnancy }\end{array}$} & No exposure & $58.1(97 / 167)$ & Reference & & Reference & \\
\hline & Medium dose ${ }^{a}$ & $69.6(16 / 23)$ & $1.65,0.64-4.22$ & 0.297 & $1.68,0.54-5.25$ & 0.375 \\
\hline & Highest dose $^{b}$ & $72.7(8 / 11)$ & $1.92,0.49-7.51$ & 0.346 & $0.98,0.20-4.84$ & 0.980 \\
\hline & & & \multicolumn{2}{|c|}{$p$ for trend $=0.173$} & \multicolumn{2}{|c|}{$p$ for trend $=0.263$} \\
\hline \multirow{5}{*}{$\begin{array}{l}\text { Household smoking } \\
\text { after birth }\end{array}$} & No exposure & $54.2(78 / 144)$ & Reference & & Reference & \\
\hline & Medium dose $\mathrm{e}^{\mathrm{a}}$ & $68.2(15 / 22)$ & $1.81,0.70-4.71$ & 0.222 & $1.29,0.43-3.90$ & 0.656 \\
\hline & Highest dose $^{b}$ & $80.0(28 / 35)$ & $3.39,1.39-8.25$ & 0.007 & $3.19,1.08-9.44$ & 0.036 \\
\hline & & & \multicolumn{2}{|c|}{$p$ for trend $=0.004$} & \multicolumn{2}{|c|}{$p$ for trend $=0.019$} \\
\hline & Total & $60.2(121 / 201)$ & & & & \\
\hline
\end{tabular}

Category of questionnaire: a 'sometimes and ceased partway' and ' 'every day'.

Table 5. Prevalence of early tooth eruption (ETE) and odds ratios (ORs) and 95\% confidence intervals (CIs) of ETE prevalence according to exposure during pregnancy and or exposure after birth including the category of no exposure to both exposure types as reference

\begin{tabular}{|c|c|c|c|c|c|}
\hline \multirow[b]{2}{*}{ Exposure type } & \multirow{2}{*}{$\begin{array}{c}\text { ETE \% } \\
\text { (n total n) }\end{array}$} & \multicolumn{2}{|c|}{ Crude } & \multicolumn{2}{|c|}{ Adjusted } \\
\hline & & OR, $95 \% \mathrm{CI}$ & $p$ & OR, $95^{\circ} \% \mathrm{CI}$ & $p$ \\
\hline No exposure ${ }^{a}$ & $61.0(150 / 246)$ & Reference & & Reference & \\
\hline During pregnancy only & $68.4(13 / 19)$ & $1.39,0.51-3.77$ & 0.522 & $1.54,0.53-4.45$ & 0.425 \\
\hline After birth only & $79.3(65 / 82)$ & $2.45,1.35-4.42$ & 0.003 & $2.29,1.19-4.40$ & 0.013 \\
\hline Both types of exposure ${ }^{b}$ & $70.7(29 / 41)$ & $1.55,0.75-3.18$ & 0.235 & $1.71,0.76-3.85$ & 0.196 \\
\hline
\end{tabular}

ETE prevalence obtained in the sensitivity analyses of the models for maternal smoking exposure during pregnancy and/or household smoking exposure after birth. ETE prevalence in the category of exposure after birth only was the highest (79.3\%) among the four exposure groups. The AOR and 95\% CI were 2.29 and 1.19-4.40 ( $p=0.013)$. ETE prevalence was higher in children in the exposure during pregnancy only $(68.4 \%)$ and both types of exposure $(70.7 \%)$ categories than in those in the reference category $(61.0 \%)$, although the differences were not significant ( $\mathrm{p}=0.425$ and 0.196 , respectively).

\section{DISCUSSION}

ETE prevalence was greater by $16 \%$ points among children exposed to household smoking every day after birth than among those who were not exposed. The association was independent of potential confounders, including maternal smoking exposure during pregnancy, and a dose-response relationship was observed. Although the observed association is represented by canines in the study, these results suggest that children who are exposed to SHS after birth have a greater chance of contact between premature enamel crystals and acid produced by fermenting oral bacteria as the basic etiology of dental caries.

The association between ETE and SHS exposure during pregnancy was consistently insignificant, although ETE prevalence was greater by $11.4 \%$ points among children who were exposed to maternal smoking every day than among those who were not exposed. The trend in ETE prevalence was unexpectedly different with regard to the subgroups for SHS exposure during pregnancy (Table 4). Reasons for the lack of significance may include the inadequate sample size of this subgroup. AOR of ETE prevalence for the medium-dose exposure 
was less than one (Table 3). Furthermore, children whose mother ceased smoking partway during the pregnancy exhibited an even lower ETE prevalence than the reference $(62.5 \%$ vs $65.5 \%$, data not shown in the results section). A similar trend in the role of exposure timing was reported for growth after birth. Maternal smoking exposure in the second and third trimester predicted an accelerated conditional weight-for-length gain by 2 years of age ${ }^{20}$. The association between maternal smoking during the first trimester and dental eruption time was not significant ${ }^{17}$. Therefore, inadequate data regarding the timing of exposure during pregnancy may be added to the reasons for the lack of significance.

The role of gestational age in ETE based on chronological age may be a third reason for the lack of significance. Because this study focused on the role of ETE in the etiology of dental caries with respect to the timing of contact between the tooth surface and acid, gestational age was not available for analyses in this study. Delayed eruption with respect to chronological age is accounted for by premature birth (i.e. shortened gestation in children with very low birthweight $)^{21}$, and may not be related to delayed dental development ${ }^{22}$. Because the causal association between maternal smoking and preterm delivery and shortened gestation was apparent ${ }^{4}$, the association between SHS exposure during pregnancy and ETE may be underestimated in children with a shorter gestational age. Furthermore, birth weight must be analyzed in relation to gestational age in weeks. Further studies that involve variables such as timing of exposure during pregnancy and gestational age are required to test the association between ETE and SHS exposure during pregnancy in detail.

A causal association of SHS exposure after birth with ETE should be carefully assessed in addition to the dose-response relationship, which was consistently significant for SHS exposure after birth. The biological plausibility is responsible for a causal inference. SHS exposure may influence the apoptosis of surrounding tissues in the alveolar bone adjacent to the coronary part of the erupting tooth $^{23}$. Tobacco constituents enhance apoptosis in periodontal tissue ${ }^{24-26}$. Thus, the space generated by cell death allows the tooth to move toward the eruption site, resulting in ETE. Another theory is derived from the role of SHS exposure in catch-up growth or in the compensatory acceleration of growth rate $^{27}$. This pathway is only applicable to the effect of exposure during pregnancy, whereas the association of a greater ETE prevalence with maternal smoking during pregnancy was not statistically significant.

Only small ${ }^{16,18}$ or no ${ }^{17}$ effects of SHS exposure during pregnancy on ETE were previously reported, wherein the association was adjusted for birth weight. Differences in ETE prevalence with regard to the exposure group were distinct in lower birth-weight $(<3000 \mathrm{~g})$ children, but the association between ETE prevalence and SHS exposure was not significant in children with a higher birth weight ( $\geqslant 3000 \mathrm{~g})$. Therefore, stratification by birth weight would be an important strategy to assess the association between ETE and SHS exposure.

We did not use a criterion in accordance with the guidelines set in force by the WHO, for example, $\leqslant 2499 \mathrm{~g}$ corresponds to a low birth weight. This criterion was developed based on epidemiological findings regarding risk of infant death ${ }^{28}$. Children with low birth weight by this criterion are relatively few in Japan. The role of SHS exposure after birth on ETE in children with birth weight $<3000 \mathrm{~g}$ may have impact on many children. Completion of primary teeth is likely correlated with body height and body mass $^{29}$. Therefore, morphological measures may be considered for confounding variables to estimate the association between SHS exposure and timing of tooth eruption of children.

The strengths of this study are that children were surveyed at a particular age with two SHS exposure types, namely during pregnancy and after birth, and that children were stratified by birth weight. However, the interpretation of the association was beyond causal inference because of the cross-sectional design. Another limitation is that insufficient data regarding the dose were available to assess the doseresponse relationship and the timing of exposure to assess the susceptible period of exposure for ETE to occur. Because the subjects in this study were not representative of Japanese children in the general population, the findings may not be generalized.

Exposure to nicotine may enhance the pathogenicity of cariogenic oral bacteria such as Streptococcus mutans ${ }^{11,30}$. Unhealthy oral behaviors such as excessive sugar intake, poor oral hygiene, and infrequent dental visits are very common in children 
who are exposed to smoking ${ }^{31}$. Therefore, cariogenic contact between bacterially produced acid and the hard enamel of tooth surfaces may be facilitated by observed ETE and accelerated by SHS exposure after birth.

\section{CONCLUSIONS}

Household smoking exposure after birth was independently associated with ETE of deciduous canines, and a dose-response relationship was observed. These results suggest that SHS exposure after birth predicts the increased probability of contact between premature enamel crystals of erupting deciduous teeth and acid produced by fermenting oral bacteria as the basic etiology of dental caries. Our findings warrant further study to examine whether ETE in children with SHS exposure after birth influences the incidence of early childhood caries.

\section{REFERENCES}

1 Best D, The Committee on Environmental Health, The Committee on Native American Child Health, The Committee on Adolescence. From the American Academy of Pediatrics: Technical report--Secondhand and prenatal tobacco smoke exposure. Pediatrics. 2009;124:e1017-44. doi:10.1542/peds.2009-2120

2 Vardavas CI, Hohmann C, Patelarou E, Martinez D, Henderson AJ, Granell R, et al. The independent role of prenatal and postnatal exposure to active and passive smoking on the development of early wheeze in children. Eur Respir J. 2016;48:115-24. doi:10.1183/13993003.01016-2015

3 Tanaka K, Miyake Y, Furukawa S, Arakawa M. Secondhand smoke exposure and risk of wheeze in early childhood: a prospective pregnancy birth cohort study. Tob Induc Dis. 2017;15:30. doi:10.1186/s12971-017-0138-7

4 U.S. Department of Health and Human Services. Chapter 9. Reproductive outcomes. In: The Health Consequences of Smoking-50 Years of Progress: A Report of the Surgeon General. Atlanta, GA: U.S. Department of Health and Human Services, Centers for Disease Control and Prevention, National Center for Chronic Disease Prevention and Health Promotion, Office on Smoking and Health; 2014;459-521.

5 Hanioka T, Ojima M, Tanaka K, Yamamoto M. Does secondhand smoke affect the development of dental caries in children? A systematic review. Int J Environ Res Public Health. 2011;8:1503-19. doi:10.3390/ijerph8051503

6 Bernabé E, MacRitchie H, Longbottom C, Pitts NB, Sabbah W. Birth Weight, Breastfeeding, Maternal Smoking and Caries Trajectories. J Dent Res. 2017;96:171-8. doi:10.1177/0022034516678181
7 Featherstone JD. The continuum of dental caries--evidence for a dynamic disease process. J Dent Res. 2004;83(Spec Iss C):C39-42. doi:10.1177/154405910408301s08

8 Avsar A, Topaloglu B, Hazar-Bodrumlu E. Association of passive smoking with dental development in young children. Eur J Paediatr Dent. 2013;14:215-8.

9 Chowdhury IG, Bromage TG. Effects of fetal exposure to nicotine on dental development of the laboratory rat. Anat Rec. 2000;258:397-405. doi:10.1002/(sici)10970185(20000401)258:4<397::aid-ar8>3.0.co;2-i

10 Li MY, Huang RJ, Zhou XD, Gregory RL. Role of sortase in Streptococcus mutans under the effect of nicotine. Int J Oral Sci. 2013;5:206-11. doi:10.1038/ijos.2013.86

11 Huang R, Li M, Gregory RL. Nicotine promotes Streptococcus mutans extracellular polysaccharide synthesis, cell aggregation and overall lactate dehydrogenase activity. Arch Oral Biol. 2015;60:108390. doi:10.1016/j.archoralbio.2015.04.011

12 Velló MA, Martínez-Costa C, Catalá M, Fons J, Brines J, Guijarro-Martínez R. Prenatal and neonatal risk factors for the development of enamel defects in low birth weight children. Oral Dis. 2010;16:257-62.

doi:10.1111/j.1601-0825.2009.01629.x

13 Carvalho JC. Caries process on occlusal surfaces: evolving evidence and understanding. Caries Res. 2014;48:33946. doi:10.1159/000356307

14 Tanaka K, Miyake Y, Nagata C, Furukawa S, Arakawa M. Association of prenatal exposure to maternal smoking and postnatal exposure to household smoking with dental caries in 3-year-old Japanese children. Environ Res. 2015;143(Pt A):148-53.

doi:10.1016/j.envres.2015.10.004

15 Tanaka S, Shinzawa M, Tokumasu H, Seto K, Tanaka $\mathrm{S}$, Kawakami K. Secondhand smoke and incidence of dental caries in deciduous teeth among children in Japan: population based retrospective cohort study. BMJ. 2015;351:h5397. doi:10.1136/bmj.h5397

16 Rantakallio P, Mäkinen H. The effect of maternal smoking on the timing of deciduous tooth eruption. Growth. 1983;47:122-8.

17 Aktoren O, Tuna EB, Guven Y, Gokcay G. A study on neonatal factors and eruption time of primary teeth. Community Dent Health. 2010:27;52-6.

18 Żạdzińska E, Sitek A, Rosset I. Relationship between pre-natal factors, the perinatal environment, motor development in the first year of life and the timing of first deciduous tooth emergence. Ann Hum Biol. 2016:43;2533. doi:10.3109/03014460.2015.1006140

19 Viscardi RM, Romberg E, Abrams RG. Delayed primary tooth eruption in premature infants: relationship to neonatal factors. Pediatr Dent. 1994:16;23-7.

20 Molnar DS, Rancourt D, Schlauch R, Wen X, Huestis MA, Eiden RD. Tobacco exposure and conditional weightfor-length gain by 2 years of age. J Pediatr Psychol. 2017;42:679-88. doi:10.1093/jpepsy/jsw095 
21 Seow WK, Humphrys C, Mahanonda R, Tudehope DI. Dental eruption in low birth-weight prematurely born children: a controlled study. Pediatr Dent. 1988;10:39-42.

22 Ramos SR, Gugisch RC, Fraiz FC. The influence of gestational age and birth weight of the newborn on tooth eruption. J Appl Oral Sci. 2006;14:228-32. doi:10.1590/s1678-77572006000400003

23 Kaneko H, Ogiuchi H, Shimono M. Cell death during tooth eruption in the rat: surrounding tissues of the crown. Anat Embryol (Berl). 1997;195:427-34. doi:10.1007/s004290050062

24 Kang SW, Park HJ, Ban JY, Chung JH, Chun GS, Cho JO. Effects of nicotine on apoptosis in human gingival fibroblasts. Arch Oral Biol. 2011;56:1091-7. doi:10.1016/j.archoralbio.2011.03.016

25 Lee SI, Kang KL, Shin SI, Herr Y, Lee YM, Kim EC. Endoplasmic reticulum stress modulates nicotine-induced extracellular matrix degradation in human periodontal ligament cells. J Periodontal Res. 2012;47:299-308. doi:10.1111/j.1600-0765.2011.01432.x

26 Semlali A, Chakir J, Goulet JP, Chmielewski W, Rouabhia M. Whole cigarette smoke promotes human gingival epithelial cell apoptosis and inhibits cell repair processes. J Periodontal Res. 2011;46:533-41. doi:10.1111/j.1600-0765.2011.01370.x

27 Riedel C, Schönberger K, Yang S, Koshy G, Chen YC, Gopinath B, et al. Parental smoking and childhood obesity: higher effect estimates for maternal smoking in pregnancy compared with paternal smoking--a meta-analysis. Int J Epidemiol. 2014;43:1593-606. doi:10.1093/ije/dyu150

28 Kramer MS. Determinants of low birth weight: methodological assessment and meta-analysis. Bull World Health Organ. 1987;65:663-737.

29 Zadzińska E, Nieczuja-Dwojacka J, Borowska-Sturgińska B. Primary tooth emergence in Polish children: timing, sequence and the relation between morphological and dental maturity in males and females. Anthropol Anz. 2013;70:1-13. doi:10.1127/0003-5548/2012/0235

30 Li MY, Huang RJ, Zhou XD, Gregory RL. Role of sortase in Streptococcus mutans under the effect of nicotine. Int J Oral Sci. 2013;5:206-11. doi:10.1038/ijos.2013.86

31. Williams SA, Kwan SY, Parsons S. Parental smoking practices and caries experience in pre-school children. Caries Res. 2000;34:117-22. doi:10.1159/000016578

CONFLICTS OF INTEREST Authors have completed and submitted the ICMJE Form for Disclosure of Potential Conflicts of Interest and none was reported.

FUNDING

Funding was received from a Fukuoka Dental College Grant for Education and Research and JSPS KAKENHI, Grant Numbers 24593182 and $15 K 11441$.

PROVENANCE AND PEER REVIEW

Not commissioned; externally peer reviewed 